\title{
Civilisations
}

Revue internationale d'anthropologie et de sciences

humaines

62 | 2013

Identité, culture et intimité

\section{Le rire des victimes}

Relations à plaisanterie au sein d'un univers de travail multiculturel

Amélie Deschenaux et Fabrice Clement

\section{(2) OpenEdition}

12 Journals

Édition électronique

URL : http://journals.openedition.org/civilisations/3299

DOI : 10.4000/civilisations.3299

ISSN : 2032-0442

Éditeur

Institut de sociologie de l'Université Libre de Bruxelles

\section{Édition imprimée}

Date de publication : 31 décembre 2013

Pagination : $91-108$

ISBN : 2-87263-042-2

ISSN : 0009-8140

\section{Référence électronique}

Amélie Deschenaux et Fabrice Clement, « Le rire des victimes », Civilisations [En ligne], 62 | 2013, mis en ligne le 31 décembre 2016, consulté le 10 décembre 2020. URL : http://journals.openedition.org/ civilisations/3299; DOI : https://doi.org/10.4000/civilisations.3299

(c) Tous droits réservés 


\title{
Le rire des victimes
}

\section{Relations à plaisanterie au sein d'un univers de travail multiculturel}

\author{
Amélie DESCHENAUX et Fabrice CLÉMENT
}

\begin{abstract}
Résumé : Dans la vie quotidienne, on s'attend spontanément à une réaction offensée, ou même violente, lorsqu'une personne est apostrophée au moyen de termes à forte connotation raciste (" négro », "sale youyou », etc.). Toutefois, une observation participante au sein d'un milieu ouvrier fortement interculturel nous a permis d'identifier de nombreuses situations où l'utilisation publique de stéréotypes culturels, voire racistes, déclenchaient au contraire le rire, y compris de la part des personnes qui étaient les cibles de ces blagues a priori douteuses. C'est le rire de ces "victimes " qui constitue le cœur de cet article. Nous tentons d'y montrer en quoi le fait de se lancer des «vannes» racistes, et d'y répondre par des rires, pourrait constituer un moyen de créer et maintenir une forme de communauté en dépit d'une forte hétérogénéité culturelle, répondant ainsi à une nécessité de "faire groupe " générée par les contraintes contextuelles de la vie en usine. La manipulation et l'appropriation de stéréotypes racistes propres à certains contextes multiculturels semblent ainsi participer à la régulation des rapports entre le "nous» professionnel et le "nous " d'origine, notamment par l'établissement de normes exclusivement en vigueur à l'intérieur du groupe de travail.
\end{abstract}

Mots-clés : coopération, culture ouvrière, ethnicité, humour, relations à plaisanterie, rire.

\begin{abstract}
When a person is accosted by the use of strong racist language, we would normally expect that person to be offended. However, participant observation in a highly intercultural working environment allowed us to identify many situations where the public use of cultural or racist stereotypes triggered laughter (rather than offense), even from the people who were the targets of these a priori dubious jokes. Analysis of the laughter of these "victims" forms the core of this article. We try to show how the act of making racist jokes, and responding with laughter, could be a way to create and maintain a form of community despite a strong cultural heterogeneity, responding thereby to a need to "be one of the guys" generated by the contextual constraints of factory life. Manipulation and appropriation of racist stereotypes specific to some multicultural contexts seem thereby to play a role in the regulation of relations between an "ethnic we" and a "professional we" which depends itself on the establishment of norms which are exclusively valid within the working group.
\end{abstract}

Keywords: cooperation, working-class culture, ethnicity, humor, joking relationships, laughing. 
$\mathrm{L}$

es énoncés comiques à caractère raciste illustrent bien le problème de la délimitation du risible. En effet, nombreux sont les exemples d'indignation publique à la suite de propos se voulant humoristiques, mais jugés par d'autres comme offensants en raison de leur caractère raciste. L'humoriste français Dieudonné a subi plusieurs procès à la suite de certains de ses spectacles considérés comme antisémites. Il a notamment été condamné pour incitation à la haine raciale en 2005. Jean-Paul Guerlain, célèbre nez français, a également été jugé en 2012 pour avoir déclaré en interview en 2010, au sujet de la création de son dernier parfum : "Pour une fois, je me suis mis à travailler comme un nègre. Je ne sais pas si les nègres ont toujours tellement travaillé, mais enfin... ». Enfin, l'affaire des caricatures du prophète Mahomet ${ }^{1}$, de leur parution en 2005 jusqu'à l'incendie des locaux de Charlie Hebdo en 2011 après leur édition spéciale et satirique Charia Hebdo, demeure encore présente dans nos esprits. Bien que ces affaires concernent des communautés différentes et reposent sur des supports médiatiques distincts, ce sont chaque fois des propos qui, se voulant comiques à l'origine, ont au final déclenché une forme de désapprobation voire d'indignation de la part d'une partie de la population, ne manquant ainsi pas de relancer le débat sur les limites du risible.

On n'est donc pas censé rire de tout. Fort de ce constat, l'énigme consiste dès lors à comprendre une partie des observations que nous ${ }^{2}$ avons menées sur les rires à l'usine. $\mathrm{Au}$ sein de notre terrain, fort hétérogène du point de vue des origines culturelles de sa population, il n'est en effet pas rare d'assister à des interpellations ou à des énoncés humoristiques à caractère raciste qui sont non seulement tolérés mais carrément accueillis par des rires, y compris de la part des victimes directes de la blague. En fait, s'offusquer de telles plaisanteries serait mal perçu, tant celles-ci semblent non seulement autorisées mais également bienvenues!

Pour tenter d'éclaircir cette énigme, nous commencerons par décrire l'univers spécifique à notre terrain en présentant la nature particulière de ce travail en usine, sa population multiculturelle et les cercles d'affinité qui y sont liés. Nous montrerons également combien ce terrain est potentiellement générateur de tensions entre collègues. Dans un second temps, nous montrerons qu'il existe malgré ces tensions un «nous collègues » transversal et englobant tous les employés de l'usine et nous insisterons sur le rôle du rire dans l'élaboration et le maintien de ce Nous. Plus précisément, ce sont les rires provoqués par les chicaneries et les blagues portant sur la délimitation d'une forme de «nous collègues » que nous mettrons en évidence. Notre hypothèse est que

1 Pour une analyse anthropologique fine de l'affaire des caricatures de Mahomet, voir Favret-Saada, Jeanne, 2007. Comment produire une crise mondiale avec douze petits dessins. Paris : Les Prairies ordinaires.

2 Si nous recourons à la forme plurielle de la première personne pour des raisons évidentes de lisibilité, seule Amélie Deschenaux a investigué le terrain. Elle y a travaillé à temps partiel durant cinq ans dans l'équipe de temporaires engagés essentiellement pour les samedis et les périodes de vacances universitaires. Ainsi si la collecte de ses observations dirigée sur les rires s'est étendue sur une période de six mois, son investigation relève bien d'une observation de type participant de longue durée, en termes ethnographiques. Elle a ainsi eu l'occasion de s'intégrer réellement à l'équipe avec toutes les familiarisations que cela implique au niveau humain, du travail, des techniques corporelles, du vocabulaire etc. 
la constitution de ce « nous collègues » est un préalable nécessaire à l'émergence du phénomène qui nous intéresse, soit l'autorisation, l'acceptation, voire l'encouragement des blagues à caractère raciste. Après avoir rapproché ces blagues des relations à plaisanterie, nous terminerons notre analyse par des considérations plus globales sur le rôle du rire dans la « fabrication » du lien social.

\section{Une poudrière dans un congélateur}

Notre lieu d'investigation est une centrale de distribution de produits surgelés appartenant à une grande chaîne de magasins suisses. Le travail consiste à y réceptionner des marchandises provenant de fournisseurs et centrales de production, à les stocker, les conditionner dans la cellule (entrepôt frigorifique ou sorte de « congélateur géant »), ainsi qu'à préparer les commandes des différents magasins répartis sur environ un tiers $\mathrm{du}$ territoire national. Les livraisons se font par camions frigorifiques dont les contenus sont chargés et déchargés sur des quais attenants à la cellule.

Le travail de manutentionnaire s'effectue dans cette cellule frigorifique où règne une température d'environ $-24^{\circ} \mathrm{C}$ ! Nous portons donc des vestes, pantalons, bottes, bonnets et gants spécifiquement adaptés à ces conditions extrêmes. Le travail y consiste essentiellement à préparer les commandes des différentes succursales en se déplaçant dans les rayons de la centrale au moyen d'une machine motorisée à l'arrière de laquelle sont empilées les marchandises, ou colis (on appelle cette partie du travail le picking). Si les déplacements entre les sections, ou zones, qui divisent les couloirs se font debout au volant de la machine, chaque chargement de marchandise nécessite bien entendu d'en descendre, de se saisir des produits sur les palettes et dans les caisses et de les empiler de façon stable et ordonnée sur les trois rolls (sortes de " caddies géants ») à l'arrière de la machine. Ces rolls sont ensuite réceptionnés par d'autres employés qui les regroupent en fonction des différentes tournées des camions.

Les marchandises à charger peuvent être lourdes et elles sont surtout nombreuses. Au-delà du froid, c'est un travail physiquement exigeant : marcher, descendre et monter, soulever, porter, charger, ranger... Les performances (nombre de colis chargés à l'heure) de chaque employé étant mesurées et contrôlées individuellement, il leur arrive de se faire avertir par un supérieur hiérarchique s'ils enchaînent les mauvaises journées au niveau de la productivité. Si l'efficacité du travailleur est le gage principal de bonnes performances, d'autres paramètres entrent également en jeu. Par exemple, en fonction du nombre de colis à charger depuis une palette, du nombre de machines à l'attente dans une section du couloir, ou encore du nombre de marchandises restant à charger jusqu'à la fin du tour, il y a certaines stratégies qu'il est opportun d'adopter afin d'améliorer ses performances. Ainsi, si la règle est de ne pas dépasser dans les couloirs, un employé qui constate que le nombre de colis qu'il doit charger dans la zone est particulièrement important peut décider de laisser passer un autre employé si ce dernier n'a qu'un très petit nombre de marchandises à charger. En ne suivant pas la consigne de ne pas dépasser à la lettre, l'employé arrivé en premier fait gagner, à un moindre coût, beaucoup de temps au suivant. Il se peut également qu'un employé soit

3 En italique dans le texte : les termes de vocabulaire propres à notre terrain ainsi que les transcriptions des occurrences de rires observées, telles qu'elles ont pu être prises en note sur le terrain. 
déjà engagé dans sa zone, procédant au chargement d'un nombre important de colis. L'employé qui arrive ensuite doit attendre que son collègue atteigne la fin de la zone. Deux choix s'offrent alors à lui : rester en retrait et en profiter pour se reposer un peu ou, au contraire, aller aider son collègue à charger ses colis. La deuxième option s'avère certes plus pénible mais elle permet aux deux employés de gagner du temps. Ces différentes stratégies nécessitent de la coopération puisque chaque employé se retrouve tour à tour dans la position de celui auquel on offre son aide, ou auquel on la refuse, et dans la position de celui qui rend la pareille. Un tel climat est évidemment générateur de tensions ; la proximité corporelle, la nécessité de remplir certaines conditions de rendement, la pénibilité du travail physique, le froid, tout concourt à provoquer quelques dérapages (insultes, bagarres entre employés). Pourtant, comme on l'a vu, il est quasiment indispensable d'entretenir des relations positives avec ses collègues.

Les personnes qui travaillent à temps plein sont tous des hommes ${ }^{4}$ âgés de vingt à cinquante ans environ, issus d'origines nationales diverses (Angola, Congo, Espagne, Erythrée, Ethiopie, Ghana, Italie, Macédoine, Maroc, Portugal, Serbie, Suisse, Tunisie, Turquie, ...). Il y a d'ailleurs tant d'origines diverses qu'il n'y a pas de groupes fortement majoritaires, exception faite des Portugais et des Noirs ${ }^{5}$. Les cercles d'affinité sont par contre très marqués par les appartenances culturelles, notamment pour ces deux groupes. Les Portugais et les Noirs entretiennent respectivement des relations étroites entre eux. Ils passent beaucoup de temps ensemble, se parlent dans leur langue d'origine, se fréquentent en dehors du travail, etc. Ces cercles sont si marqués qu'ils ont une influence directe sur la répartition de la population de l'usine dans les locaux de pause : les Africains dans le local non-fumeur, les Portugais dans le local fumeur. Et ce n'est pas la cigarette à elle seule qui justifie cette répartition puisque plusieurs Portugais ne fument pas du tout, alors que certains Africains font au contraire l'allerretour entre les deux locaux afin de fumer d'un côté et de passer le reste du temps de pause de l'autre!

\section{Nous (ouvriers) et les autres}

Les activités professionnelles de notre usine impliquent des cercles d'affinités divers et particulièrement saillants. Les tensions liées à la difficulté de la tâche nous forcent à nous interroger sur la manière dont ces petits « nous d'origine » parviennent à cohabiter au sein d'un contexte potentiellement explosif. Une piste de réflexion nous est offerte par les travaux de sciences sociales portant sur différents milieux professionnels, y compris ouvriers ; ceux-ci ont en effet mis en évidence l'importance du rire dans ce type de situations. Ce constat général de Frisch-Gauthier s'applique par exemple parfaitement à notre investigation empirique :

4 Seules deux femmes sont employées en cellule en tant que temporaire, à temps partiel.

5 Note sur l'usage du terme « Noir » : il s'agit ici évidemment de la catégorie locale, en l'occurrence de la terminologie mobilisée par les employés de la centrale. Bien que de nationalités différentes (Angola, Congo, Erythrée, Ethiopie, Ghana), les Africains à la peau noire sont désignés par le terme de Noir, aussi bien par leurs collègues que par eux-mêmes. 
Enfin presque le quart de nos plaisanteries consistent en mimiques, en signes ou en bruits. Ce caractère non verbal caractériserait certainement une bonne partie des échanges qui interviennent dans le cadre de l'atelier. L'importance de ce mode de communication s'explique par le bruit qui gêne les échanges verbaux, par la distance qui sépare parfois les postes de travail, mais aussi par la plus grande aisance des ouvriers en ce domaine qu'en celui de la parole. Le trait d'esprit, au sens classique du terme, n'est pas tellement fréquent en milieu ouvrier. Les images, les gestes, les situations concrètes fournissent un matériel qu'on y utilise plus volontiers. (Frisch-Gauthier $1961: 303$ )

De manière générale, nous constatons également que les rires quotidiens en usine ne sont pas issus de véritables blagues, énoncés comiques ou autre locutions humoristiques. Ils relèvent plutôt de " chicaneries », même si ces dernières n'épuisent pas les types de rire que l'on retrouve dans notre corpus de données. Notre lieu de travail, la cellule frigorifique, n'est en effet pas propice aux échanges verbaux ; le bruit des machines, des souffleries, les bonnets et écharpes couvrant les oreilles ainsi que les déplacements en solitaire sur sa propre machine rendent les échanges verbaux entre collègues peu fréquents, peu aisés et brefs. Et pourtant, les rires ne manquent pas d'y retentir !

En effet, un phénomène qui appartient plutôt au monde de l'enfance y est régulièrement observé : des rires bruyants sont fréquemment provoqués par des formes de bagarres amicales. Entre collègues, on se saute dessus, on s'empoigne, on court en faisant mine de se charger et on se frappe plus ou moins gentiment ... Les rires sont particulièrement prononcés chez les participants directs à la « bagarre » mais, en principe, la plupart des collègues assistent à la scène en rigolant, ou au moins en souriant. Pour rendre compte de ces éclats de violence douce, rappelons le cadre de ce travail qui contraint les employés à une certaine proximité physique. Les ouvriers à plein temps travaillent dans la même cellule, et toutes les $1 \mathrm{~h} 40$ passent ensemble une pause de 20 min dans des locaux à température ambiante afin de se réchauffer. Ces conditions particulières enjoignent les employés à relativement bien se connaître. De plus, le fait de travailler toute l'année à $-24^{\circ} \mathrm{C}$ en tenue « polaire » fait de ces ouvriers une catégorie bien particulière et reconnaissable de travailleurs. Ainsi, le rire de chicanerie que nous avons observé semble se produire dans le cadre de ce que nous proposons de nommer une « double proximité » : une proximité physique extrême, d'une part, puisqu'il y a contact physique au sens propre et, d'autre part, une proximité humaine impliquant une forme de bonne entente, voire de rapports amicaux. Le caractère particulier de ces échanges quotidiens mène en effet ces partenaires à partager une certaine forme d'intimité. Cette proximité entre ouvriers a été mise en évidence par Parry (2002). Selon lui, sur le terrain indien, de tels liens se créent et se maintiennent en dépit de différences interindividuelles de castes, d'origines et de religions. Dans notre cas, les chicaneries n'ont ainsi pas leur place entre inconnu-e-s : c'est en tant que marque de cette " double proximité » que le rire de chicanerie signale une forme d'appartenance de groupe. On ne se chicane qu'entre individus particulièrement proches. Les membres $\mathrm{du}$ 《 nous collègues » constituent ainsi un cercle spécifique, une sphère semi-privée qui se met en place en dépit d'une forte hétérogénéité au niveau des origines de ses membres.

Ces chicaneries, bousculades et bagarres « pour de faux » peuvent être assimilées à ce que Dunbar appelle l'épouillage social (« social grooming ») associant contact et 
sensation de type proprement physique et contact et sensation de type social (Dunbar 1996 : 1). Sa thèse globale est que, du point de vue de l'évolution, les sociétés humaines ont progressivement remplacé la pratique de l'épouillage par les échanges langagiers. Selon lui, la taille trop importante des groupes dans lesquels vivent les êtres humains les aurait poussés à remplacer l'épouillage, trop coûteux en temps, par le langage. On n'observe ainsi généralement que peu de chicaneries entre adultes humains, sauf dans des conditions rares et très spécifiques de camaraderie, comme au sein d'une équipe de sport par exemple. En plus de la « double proximité », la taille restreinte de notre groupe d'ouvriers pourrait ainsi bel et bien favoriser l'émergence de chicaneries entre adultes. Comme l'épouillage simiesque et le langage humain, les chicaneries et rires qui en découlent participent ainsi directement de la création et du maintien du lien social.

En dehors de ces rires liés à des chicaneries, certains rires provoqués par des blagues, qui ont plutôt lieu dans les locaux de pause pour les raisons invoquées plus haut, témoignent également de l'élaboration quotidienne d'un «nous collègues ». Et un des moyens les plus efficaces de rendre l'unité d'un groupe saillante est d'y opposer un groupe dont les caractéristiques diffèrent fortement des siennes. Dans notre cas, c'est une distinction entre des collègues de la même entreprise mais travaillant à des postes et en des lieux différents qui est mobilisée. En effet, si notre équipe est composée de manutentionnaires en cellule frigorifique, elle doit tout de même parfois interagir avec des collègues qui travaillent « au chaud» comme employés de bureau.

Dans les couloirs pendant la pause, nous bavardons avec une collègue du bureau. Martine est une des responsables de la gestion informatique du système qui gère le travail au sein de ce congélateur géant. Nous parlons bien entendu du boulot et plus particulièrement des pannes de système. Elle s'exclame alors :

\section{- "y a déjà plus de pannes à cause de Martine [elle-même], c'est déjà ça!"» - «ça fait déjà bien longtemps! » [moi] \\ - « attends, parle pas trop vite j'travaille le samedi de Pâques!»}

Cette réflexion provoque un éclat de rire partagé et simultané. Remarque : Martine fait référence aux nombreuses pannes rencontrées au moment où elle apprenait à gérer le système informatique et qu'elle se retrouvait seule aux commandes le week-end. C'était même devenu une blague récurrente ; nous savions que nous finirions plus tard lorsque c'était elle qui occupait ce poste ! Même si cela fait effectivement longtemps que cela ne s'est pas produit et qu'elle maitrise désormais parfaitement son travail, elle fait ironiquement référence au samedi de Pâques car, entouré de jours fériés, il coupe un long week-end où personne n'a envie de travailler!

Ce rire rend saillant le groupe de collègues de manière indirecte. L'allusion au risque de panne met en exergue une distinction d'appartenance. En effet, la destinée des ouvriers repose en partie sur la responsabilité de Martine, qui travaille en bureau. Les employés du bureau ont d'ailleurs une grande responsabilité quant au niveau du déroulement de chaque journée de travail ; une erreur de leur part peut influencer la journée de chacun des employés en cellule. C'est cette distinction en terme de responsabilité qui rend visible l'appartenance au « nous collègues du froid » par rapport aux collègues indirects du bureau, travaillant « au chaud». Si la réflexion de Martine met en évidence la distinction existant entre travailleurs du froid et employés de bureau, 
le fait que les deux protagonistes en rigolent indique également et paradoxalement une forme de proximité et de lien entre les deux statuts. En effet, le temps de travail de Martine dépend également de ce qui se passe en cellule ; elle ne peut quitter son poste informatique qu'une fois toutes les commandes de la journée préparées. Ainsi, si son degré de responsabilité est rendu saillant par la plaisanterie, celle-ci souligne par la même occasion que bien qu'au chaud, Martine subira les mêmes conséquences que les employés de la cellule au niveau de son horaire de travail. La plaisanterie et les rires qui en découlent indiquent aussi bien une distinction hiérarchique qu'une forme de symétrie par rapport aux horaires de travail.

L'occurrence suivante relève du même procédé dichotomique (chaud/froid, bureau/ cellule, confortable/pénible, responsabilités/conséquences), mettant en évidence cette fois non la distinction de responsabilité mais le degré de pénibilité du travail.

\begin{abstract}
Dans les couloirs pendant la pause, discussion avec Pierre qui travaille au bureau. Je lui demande comment se porte sa tendinite au tendon d'Achille. Il me dit alors qu'il doit faire de la physio et que les exercices lui font bien mal et qu'il est censé les faire régulièrement. Je lui demande s'il les fait, il me répond en souriant qu'il n'a pas le temps. Je lui réponds « t'es gonflé j' suis sûre que tu peux même les faire pendant les heures d' boulot... ». Nous éclatons de rire simultanément, Pierre me répondant « c'est vrai ! ». Une collègue de bureau de Pierre rigole également.
\end{abstract}

La dernière réflexion tenue est volontairement ironique. En effet, Pierre travaille assis dans son bureau, devant un ordinateur, pendant que nous sommes en train de nous affairer dans une cellule frigorifique ! Souligner cette distinction par l'ironie permet de signaler la différence de statut entre le groupe de travailleurs à l'usine et ceux, dont fait partie Pierre, qui travaillent au bureau. Dans ce cas, comme dans l'exemple précédent, la référence commune repose sur les statuts respectifs des protagonistes : celui d'employé $\mathrm{du}$ froid et celui d'employé de bureau. Signaler ironiquement à Pierre que son travail est moins pénible que le nôtre revient à l'exclure de ce groupe d'appartenance précis et, par là, à renforcer celui-ci en mettant en évidence une de ses caractéristiques. C'est la référence conjointe à un statut professionnel - ouvrier ou administratif - qui permet la constitution de deux groupes d'appartenance relativement exclusifs.

Ce qui nous intéresse surtout dans ce type d'occurrence, c'est la référence à des conditions communes de pénibilité. En tant que collègues, nous partageons tous le même espace, le même contexte, les mêmes conditions de travail, de salaire, le même savoir-faire. Mettre ironiquement en évidence qu'un collègue de bureau a le loisir de faire ses exercices de physiothérapie pendant ses heures de travail visibilise par effet d'opposition les conditions pénibles du travail en cellule. Rendre saillantes des conditions de pénibilité a pour effet de rendre saillant par extension le groupe qui les partage. Le « nous collègues du froid » existe en grande partie par les caractéristiques du travail communément partagé. La réflexion suivante s'inscrit également dans ce type de procédé.

A la pause de $9 \mathrm{~h}$, je croise Thierry dans les couloirs. Ne l'ayant pas encore vu, je lui lance : « Hey salut ! Ça va ? » Il me répond alors : « tu veux vraiment savoir? » Le ton lassé sur lequel il me répond me pousse à lui dire : « non pas vraiment en fait ! » en lui souriant. Il enchaîne alors : «c'est dommage parce que 
j'avais trouvé une bonne formule ce matin... ». Voyant qu'il a envie de le faire, je lui dis alors : « vas-y alors c'est quoi la formule ? » Il me dit alors avec un grand sourire : «j' préférerais m' couper une main plutôt que d' venir bosser... » Ce qui provoque un éclat de rire de ma part, puis de la sienne. La formule « j' préférerais m' couper une main plutôt que d' venir bosser... », au moyen d'un procédé d'exagération, met elle aussi en évidence les conditions de pénibilité partagées par tous les membres du «nous collègues du froid».

Les rires traités jusqu'ici, qu'ils soient issus de chicaneries ou d'énoncés comiques, semblent bien participer à l'élaboration, à la gestion, au maintien d'un " nous collègues » contraint par l'environnement et les conditions de travail. Il semble que la constitution de ce groupe, et c'est bien le cœur de notre propos, constitue en fait un préalable nécessaire à la circulation et à l'acceptation de blagues racistes.

\section{Un rire « réfrigérant»}

La pause de midi se passe dans un calme relatif, Azmi s'agitant au sujet de son tour pour le micro-ondes, les autres mangeant plutôt en silence. A un moment, Darko s'adresse à moi. Il s'exprime avec un fort accent macédonien mais progresse très vite dans son apprentissage du français ${ }^{6}$ et, malgré le fait qu'il y ait parfois quelques problèmes de compréhension, il fait souvent l'effort de s'exprimer, notamment relativement à des choses relatées par les médias. Il me dit alors qu'il a vu un reportage sur Hong Kong - enfin s'il s'en souvient bien selon ses dires, c'est bien de Hong Kong qu'il s'agit - à la télévision hier soir. Il me dit « C'est 30 millions d'habitants...c'est quinze fois comme la Macédoine...y a deux millions dans toute la Macédoine ! ». Jean lui dit alors. « Ah ouais deux millions quand même ? ! J'voyais ça encore plus petit ", à la suite de quoi, Raimundo enchaîne sur un ton tout à fait sérieux : « Deux millions...c'est déjà un million et demi de trop!». Cette réflexion nous fait rire, Darko et moi, ce qui provoque un grand sourire satisfait de Raimundo.

La réflexion «c'est déjà un million et demi de trop ! » est lancée par un collègue portugais à l'adresse d'un collègue macédonien au sujet du nombre de ses compatriotes. Il s'agit clairement d'une vanne raciste sous-entendant que Raimundo ne manifeste que peu de considération pour les habitants de la patrie d'origine de Darko. Il semblerait donc légitime de s'attendre à une réaction offensée de la part de la personne victime de ce type de réflexion, et pourtant, dans ce cas précis, Darko éclate de rire.

Ce rire collectif est pour le moins surprenant : non seulement il comprend des traits d'humour à fortes connotations racistes mais les personnes dont « on rit» rient à leur tour. Notre terrain présente en effet la particularité de nous avoir permis d'observer nombre de situations où l'utilisation publique de stéréotypes culturels, voire racistes, déclenchait le rire, y compris de la part des personnes qui étaient les cibles de ces plaisanteries a priori douteuses. C'est précisément ce rire énigmatique que cet article vise à mieux comprendre.

6 Il faut noter que les employés d'origine non-francophone apprennent la langue, pour la plupart d'entre eux, de manière toute phonétique, sans jamais prendre de cours. Certains conservent des accents très prononcés, voire des formulations difficiles à saisir pour des non-initiés. 
Local de pause fumeur. Nizar, un collègue Tunisien qui feuillette le quotidien « La Liberté » ${ }^{7}$ du 7 avril 2010 attire l'attention d'Alexandre, son collègue suisse assis à côté de lui, sur un des gros titres ; «t'as vu ça ? ! ?». Le titre indique qu'une femme, mère de deux enfants, s'est faite poignardée et égorgée par son mari dans le quartier du Schoenberg à Fribourg. Alexandre jette un œil et Nizar continue de lire. Tout d'un coup, il s'exclame : " Noooon!? C'est un Tunisien !». La réflexion provoque quelques sourires en coin d'autres collègues et une réflexion : «ça t'étonne? ». Nizar poursuit alors, « tu lis l' titre, tu t' dis, ça c'est un Noir... pis non c'est un Tunisien!» Cette réflexion provoque l'éclat de rire de plusieurs collègues présents, dont je fais partie.

L'épisode de rire que déclenche cet énoncé « comico-raciste » désigne, à l'aide de « critères raciaux », deux groupes ou collectifs : Tunisiens et Noirs. Nizar, en faisant la réflexion «Tu lis l'titre, tu t'dis, ça c'est un Noir...pis non c'est un Tunisien ! », s'inscrit publiquement dans le premier groupe, par opposition au second. Ce type d'énoncé au caractère transgressif révèle une forme d'accord tacite partagé entre les rieurs. En effet, le fait de rire à une réflexion raciste revient à accepter sa dimension transgressive ; dans notre contexte, rire à une telle réflexion ne signifiant pas véritablement être raciste. Le public prend donc l'énoncé avec humour, alors qu'il pourrait tout aussi bien s'en offusquer ou s'en choquer. A noter que l'un des collègues assis juste à côté de Nizar, et qui lui aussi rit à la suite de sa réflexion, est justement noir, il fait partie des fumeurs qui s'en vont dans l'autre local à peine leur cigarette consumée. Comme dans le cas de la réflexion « anti-Macédoniens », la victime de la locution raciste la plébiscite en rigolant au lieu de s'en offusquer.

Dans le contexte de notre investigation, le fait de rendre visible, voire de moquer ou insulter des groupes culturels semble à la fois courant et non problématique, y compris pour les victimes de ces réflexions. Il est intéressant de noter que les identités culturelles que les plaisanteries rendent saillantes varient fréquemment. Si les appartenances nationales sont souvent utilisées pour s'interpeller mutuellement ; « Hé Portugais/ Tunisien/Macédonien ! etc. », le recours à des groupes d'appartenance de plus grande taille est également adopté, du type « Hé Africain/Negro/Musulman/Youyou ! ». De plus, dans notre usine, l'usage de ce type d'interpellations n'est pas réservé uniquement aux membres du groupe d'appartenance. En effet, bien qu'il soit relativement commun que des amis africains s'appellent mutuellement « negro » ou que des ressortissants d'ex-Yougoslavie se qualifient réciproquement de " youyous », il est nettement moins courant que ce type d'interpellation raciste soit autorisé à une personne qui n'est pas membre du groupe en question. Or, c'est bien le cas dans notre usine ; quelle que soit son origine, un employé peut se permettre d'appeler un de ses collègues « negro »! Il semble que ce soit la forte hétérogénéité culturelle du groupe ainsi que la double proximité qui lie les employés entre eux qui favorisent ce type de phénomène. Parfois, le groupe d'identification peut même s'élargir à une forme de " nous non-suisses », caractéristique partagée par une grande partie des employés de la centrale. En effet, le fait de ne pas être de la nationalité de la patrie d'accueil constitue le point commun identitaire de nombreux employés, leurs nationalités et cultures d'origines étant, nous

7 Article payant en ligne : http://www.laliberte.ch/galeries/la-mere-de-deux-enfants-meurt-sous-lescoups-de-couteau-de-son-epoux 
l'avons dit, fortement hétérogènes. L'illustration qui suit relève spécifiquement de ce « nous non-suisses » :

Alvaro, employé portugais d'une quarantaine d'année raconte de façon récurrente une anecdote relative à son arrivée en Suisse, il y a de cela quelques années. Tous les collègues la connaissent, ce qui ne les empêche pas d'en rigoler à chaque fois. Alvaro raconte notamment cette anecdote aux nouveaux employés temporaires. Il explique comment, en arrivant en Suisse, il a contracté un important crédit auprès d'une banque du pays, ce qui, selon ses dires, aurait facilité la régularisation de ses papiers. Sa conclusion en est la suivante : si tu dois de l'argent à la Suisse, alors on va t'empêcher d'en partir!

Cette anecdote, quel qu'en soit le degré de véridicité, a une résonance particulière pour tous les employés non-suisses, confrontés aux problèmes des papiers de séjour et d'identité, ainsi qu'aux nombreuses formalités propres aux droits et obligations des étrangers. Elle ironise également sur la patrie commune d'accueil en mettant en évidence le lien entre immigration et argent. Ainsi, dans un contexte de forte hétérogénéité culturelle, la non-appartenance à un groupe peut donner lieu, par contraste, à un nouveau groupe d'appartenance.

Cet exemple, tout comme les autres plaisanteries à caractère raciste mentionnées jusqu'ici, semble indiquer une relation étroite entre le rire et le « marquage » d'une appartenance de groupe. Au sein de notre cellule frigorifique, répondre à une blague raciste par des rires pourrait constituer un moyen de créer et maintenir une forme de communauté en dépit d'une forte hétérogénéité culturelle, répondant de fait à la nécessité de « faire groupe » dans le contexte difficile de la vie en usine. En effet, la manipulation et l'appropriation de stéréotypes racistes propres à certains contextes multiculturels semblent participer à la régulation des rapports entre le «nous » professionnel et le « nous » d'origine, notamment par l'établissement de normes exclusivement en vigueur à l'intérieur du groupe des co-travailleurs. Les nombreuses observations, auxquelles nous incluons nos propres éclats de rire, accumulées lors de cette vie en commun au sein de l'usine mettent en évidence la négociation progressive et quotidienne de l'élaboration d'un «nous » professionnel malgré des différences culturelles manifestes.

Notre hypothèse est que, dans les situations de travail en milieu interculturel, les rires qui ponctuent ces blagues racistes résultent des tensions induites par le " frottement » entre des habitus culturels différents - tensions qui doivent être impérativement désamorcées au sein de ce contexte professionnel qui exige la coordination rapprochée des uns et des autres afin d'organiser le travail en commun. Ainsi, les rires, issus plus spécifiquement de " chicaneries » et de blagues racistes, participent selon nous à l'équilibrage subtil des différents « nous d'origine » mutuellement exclusifs au sein d'un « nous collègues » plus englobant.

\section{Monde du travail et " relations à plaisanterie "}

Les phénomènes mentionnés jusqu'ici, chicaneries exceptées, ne manquent pas d'évoquer les « relations à plaisanterie ${ }^{8} »$ que la tradition anthropologique a maintes fois

8 Ce que nous nommons « relations à plaisanterie » correspond au terme de Joking Relationship, également traduit - comme dans la citation ci-dessous - par «parenté à plaisanterie ». 
mises en évidence (Allès 2003 ; Brackelaire 1993 ; Diallo 2006 ; Fay 2006 ; Labouret 1929 ; Moreau 1944 ; Paulme 1939 ; Radcliffe-Brown 1968 ; Sissao 2004). RadcliffeBrown définissait à l'origine ce concept de la manière suivante :

$L a$ " parenté à plaisanteries » est une relation entre deux personnes dans laquelle l'une est autorisée par la coutume, et dans certains cas, obligée, de taquiner l'autre ou de s'en moquer ; l'autre, de son côté, ne doit pas en prendre ombrage. On en distingue deux variétés principales : dans l'une, la relation est symétrique, chacune des deux personnes se moque de l'autre; dans l'autre, la relation est asymétrique: A fait des plaisanteries aux dépens de B et B accepte la moquerie avec bonne humeur et sans y répondre; ou bien A plaisante B autant qu'il lui plaît, tandis que B ne plaisante A que très discrètement. (Radcliffe-Brown 1968 : 158)

Les occurrences de rire que nous avons observées sur notre terrain relèvent plutôt de relations à plaisanterie de type symétrique. C'est notamment le cas de la totalité des blagues à caractère raciste ; si ce genre de blague provoque le rire même de sa victime dans notre contexte c'est qu'elle fonctionne sur un rapport symétrique du type « chacun d'entre nous est l'étranger de quelqu'un d'autre ». En effet, si une multitude d'origines ethnico-culturelles se côtoient quotidiennement dans la cellule, il faut préciser que les Suisses y constituent une minorité au même titre que les autres nationalités. Cette hétérogénéité permet une forme de réciprocité : il est possible de se moquer d'un Portugais en étant Macédonien puisque l'inverse se produit également. On se taquine ainsi de façon symétrique sur nos origines respectives.

Les relations à plaisanterie que l'on qualifierait d'asymétriques concernent plutôt les blagues qui marquent la distinction entre collègues du froid et du bureau. S'il y a bien une asymétrie hiérarchique de nature entre la position de manutentionnaire en cellule frigorifique et celle d'employé de bureau, elle se manifeste en miroir dans les blagues qui y font référence. S'il est en effet possible pour un employé de la cellule de se moquer ouvertement d'un collègue de bureau, c'est en référence directe à cette asymétrie ; le travail de bureau est moins pénible et il implique potentiellement des conséquences directes sur le travail au froid, alors que l'inverse n'est pas vrai.

Au-delà des structures types des relations à plaisanterie qu'il observe, RadcliffeBrown s'intéresse aux spécificités des relations entretenues entre les groupes qui y recourent. Et s'il met quant à lui (1968 : 201) en évidence les relations d'opposition, entre grands-parents et petits-enfants d'un côté et leurs enfants et parents respectifs de l'autre, caractérisant l'émergence de relations à plaisanterie dans les tribus estafricaines qu'il observe, les groupes d'opposition que nous observons pour notre part relèvent d'affinités notamment liées à la culture d'origine, ainsi que de relations de travail. Toutes ces relations à plaisanterie participent ainsi de la régulation de différents nous, s'opposant et s'incluant à la fois les uns les autres. Le «nous collègues du froid » s'oppose ainsi à tout autre groupe de travailleur du chaud, y compris à celui de la même usine, alors même qu'ils sont unis par leur travail respectifs et les horaires qui y sont directement liés. Les « nous d'origine » s'opposent les uns aux autres de façon exclusive à un premier niveau (nous-Portugais, nous-Noirs, nous-Tunisiens, nousMacédoniens etc.) mais se retrouvent en partie inclus dans un "nous non-Suisses » plus vaste s'opposant aux quelques employés de la centrale n'ayant aucune origine 
étrangère. Enfin, tous ces divers « nous d'origine » sont inclus dans le même « nous collègues du froid $»^{9}$.

Ce type de plaisanteries semble jouer un rôle de pacificateur des relations sociales en balisant les situations où les rires sont « permis », voire socialement encouragés. En favorisant les plaisanteries dans des situations où la nature des relations sociales et de la répartition du pouvoir suscitent de fortes tensions interindividuelles, l'institution de relations de plaisanterie assure une forme de catharsis et de décontraction des relations sociales. Radcliffe-Brown met par ailleurs en évidence la spécificité du contexte nécessaire à l'émergence de ce phénomène :

La parenté à plaisanteries est une combinaison singulière de bienveillance et d'antagonisme.Dans tout autre contexte social, ce comportement exprimerait et éveillerait l'hostilité ; en réalité, il ne signifie rien de sérieux et ne doit pas être pris comme tel ${ }^{10}$. Cette hostilité apparente est la contrepartie d'une amitié réelle. Autrement dit, la relation implique la permission au manque de respect. (Radcliffe-Brown 1968 : 159)

Il est relativement évident que les blagues racistes éveillent l'hostilité dans la plupart des contextes. Ce n'est pourtant pas le cas dans notre usine où se côtoient quotidiennement des individus certes différents les uns des autres mais appartenant au même " nous collègues ». Bradney (1957) mobilisait d'ailleurs spécifiquement le concept anthropologique de relation à plaisanterie dans le cadre d'un milieu ouvrier. Comme elle, nous considérons que le milieu industriel de notre investigation présente une série de points communs avec le milieu des sociétés dites primitives étudiées par les anthropologues. Ce sont en effet des univers qui ont en commun de contraindre à une forme de proximité, aussi bien spatiale que fonctionnelle, afin d'assurer une collaboration optimale. Traditionnellement, il s'agissait essentiellement de relations maritales, familiales et commerciales intertribales alors que le milieu industriel implique des relations amicales entre des individus souvent issus de sous-cultures différentes. Les interactions entre ces sous-groupes impliquent une « gestion émotionnelle » où il convient de maîtriser ses propres manifestations de rires et de sourires (Hochschild 2003). En ne s'offusquant pas sur une blague raciste visant sur son groupe d'appartenance, l'ouvrier en situation de double proximité assure sa participation à la vie d'un " entre-nous " professionnel. Dans ces conditions, le rire au travail ne porte pas sur les supérieurs ou sur le règlement (Coser Laub 1960 ; Frisch-Gauthier 1961 ; Dwyer 1991 ; Lynch 2010). Il relie des individus forts différents du point de vue de leur origine placés en situation de double proximité contrainte au sein d'un même « nous collègues ». Dans ce contexte, l'humour est bien « un moyen puissant de négociation des identités, de délimitation des appartenances, de définition de l'inclusion et de l'exclusion » (Ratouis et Baumeister 2011 : 10).

9 Pour une discussion sur la notion de « Nous », voir Laurence Kaufmann 2002.

10 Nous mettons en évidence étant donné la correspondance exacte avec notre description empirique. 


\section{Pourquoi le rire, pourquoi en rire?}

$\mathrm{Au}$ terme de ce travail descriptif, nous aimerions tenter de comprendre pourquoi le rire semble être si fréquemment recruté lors de situations où des activités sociales qui peuvent à tout moment susciter le conflit doivent, pour l'intérêt de chacun, se poursuivre de manière plus ou moins harmonieuse. Dans cette perspective, nous proposons d'envisager les situations de travail décrites ci-dessus comme des formes particulières d'interactions sociales; pour effectuer ces activités de concert, il est indispensable que les individus coopèrent, même si leurs intérêts personnels immédiats peuvent différer. Or ce « mystère » de la coopération humaine a récemment donné lieu à de nombreuses recherches inspirées par des modèles biologiques.

Commençons par décrire la situation sociale complexe dans laquelle se trouvent les employés de la cellule. D'une part, leur travail est rude, a priori individuel, mais de nombreuses formes de coopération émergent au cours de la journée, coups de main occasionnels ou réguliers qui ont pour effet d'alléger la tâche des employés. Pour qu'un tel système d'aides informelles persiste à travers le temps, il faut donc que chacun « joue le jeu ». D'un point de vue expérimental, il a été démontré que les individus humains sont très sensibles à la rupture de telles normes sociales d'équité et qu'ils sont mêmes prêts à investir une part de leur bénéfice pour s'assurer que les free-riders soient punis (Fehr et Fischbacher 2004 ; Fehr et Gintis 2007). Parallèlement à cette dimension punitive, nous semblons être très habiles à détecter dans les interactions quotidiennes les signaux qui indiquent un alignement des comportements. Ainsi, nous nous sentirons à l'aise avec ceux qui partagent les mêmes normes interactionnelles (signes de politesse, manière de s'exprimer, etc.) et qui partagent les mêmes manières de juger comme « valable » un certain style interactionnel (Gil-White 2005). Généralement, ces manières d'être et d'agir constituent précisément une des dimensions propres à la culture dans laquelle les individus ont été socialisés.

C'est sur ces indices d'appartenance sociale - notamment les alignements comportements qui découlent du partage des mêmes ethos corporels - qu'une préférence dans l'attribution des ressources et une certaine tolérance pour la tricherie se dessinent entre ceux qui appartiennent au groupe ( « in-group ») et ceux qui lui sont extérieur (« out-group ») (Bernhard, Fischbacher et Fehr 2006). Or, dans les situations de travail décrites jusqu'ici, la plupart des facilitateurs culturels qui, en principe, favorisent l'interaction et la coopération, sont absents. Les employés proviennent non seulement de régions diverses mais de pays et de cultures très différents. On peut donc s'attendre à ce que les codes implicites de communication soient, du moins dans un premier temps, difficiles à interpréter pour ces travailleurs. De plus, les conditions de travail sont dures et, à cause du froid, ils doivent tous partager, à intervalle régulier, des pauses au sein d'un local de taille assez réduite à température ambiante afin de réchauffer leurs organismes. Bref, tout est « fait » pour que les rapports sociaux ne se déroulent pas sans anicroches. La seule différence par rapport à une situation de conflit, et c'est une différence essentielle, est que tous ces individus ont un but partagé : en commun, ils doivent remplir dans les temps les camions frigorifiques qui attendent pour aller livrer leurs marchandises. En un sens, leurs interactions sont remplies de signaux qui ont toutes les chances de prétériter la coopération; mais, le risque étant grand de 
perdre son travail si le conflit s'installe, ces signaux doivent être, pour ainsi dire, mis « entre guillemets » lors des interactions quotidiennes.

C'est dans ce contexte qu'intervient le rire. D'un point de vue physiologique, le rire semble renvoyer à un phénomène individuel bien identifiable : il consiste en une segmentation d'expirations en séries de vocalisations vocales courtes qui se répètent environ chaque cinquième de seconde (Provine et Emmorey 2006 : 403). Mais ce phénomène n'est individuel qu'en apparence : comme l'a montré Provine, le rire semble renfermer une fonction spécifiquement sociale : "Mes étudiants riaient trente fois plus quand ils étaient dehors en groupe que seuls - le rire disparaissait même chez les sujets qui n'étaient pas exposés aux stimulations des médias. [...] » (Provine 2003 : 53-54). D'un point de vue physiologique, le rire est bien entendu associé à la joie, une émotion fortement positive (Panksepp 2007). Pour Hayworth, le rire fut d'abord un signal adressé aux autres membres d'un groupe afin de leur signifier le relâchement d'une tension après un danger - « une sorte de "ouf !" élaboré » (cité in Servais 1999 : 164). Le rire, on le sait, est également contagieux et il tend à déclencher la bonne humeur ; mais pas dans n'importe quelles conditions. Ainsi, les rires vocaux assez forts et chantants déclenchent chez les auditeurs une émotion positive (Bachorowski et Owren 2001). De plus, plus les membres d'un groupe deviennent amis et plus ces rires vocaux vont entraîner d'autres rires (Smoski et Bachorowski 2003). Les rires, par rapport aux sourires, seront même plus nombreux lorsque les groupes sont formés d'individus de même rang social (Mehu et Dunbar 2008). Tous ces éléments ont encouragé Owren et Bachorowski (2003) à proposer l'hypothèse selon laquelle le rire aurait évolué moins pour transmettre une certaine information sur l'état émotionnel de l'émetteur qu'en raison des effets apaisants qu'il exerce sur les états affectifs des récepteurs. Dans ce contexte, le rire aurait alors évolué parce qu'il constitue, en vertu de ses propriétés acoustiques, un remarquable mécanisme pour établir et maintenir des relations positives entre des individus en interaction.

$\mathrm{Si}$, pour les besoins de notre argumentation, nous acceptons cette passionnante hypothèse, on comprend mieux comment le rire a pu être recruté dans des situations de jeu. En riant, l'agresseur « pour rire » indique que son attaque est simulée, qu'elle ne porte pas à conséquence. En riant à son tour, l'agressé montre qu'il accepte de « jouer le jeu » et entre dans une ronde sociale de plus ou moins longue période. Les plaisanteries de la cellule constituent bien des formes d'agression et ces « vannes » racistes circulent impunément, isolant certains stéréotypes culturels qui, peut-être, renvoient à des manières d'être dont il est difficile de « faire façon ». Comme le dit Servais, les rires permettent l'élaboration d'interactions sociales proprement humaines où le jeu permet d'être à la fois hostile et non hostile, amical et non amical, soumis et insoumis (Servais 1999 : 173). En rendant possible l'expression de tensions inhérentes à toute forme d'interaction sociale, et plus encore dans des contextes fortement interculturels, on peut donc certainement admettre avec La Fontaine que « le rire est ami de l'homme ».

\section{Conclusion}

Les rires qui résonnent dans l'usine après des plaisanteries racistes ne peuvent que surprendre un observateur extérieur; dans un autre contexte, de tels énoncés aviveraient les hostilités, voire déclencheraient des altercations physiques. Or, entre les murs de la 
cellule frigorifique, ceux-là même qui sont visés par les « vannes » racistes rient de concert. Notre observation participante a permis de mieux faire sens de cette énigme psychosociologique.

En usine, les rires semblent participer à la régulation des relations de groupes à plusieurs niveaux. Ils participent à la création, au maintien, à la destruction ou encore à la gestion des différents groupes sociaux. Dans un univers où, d'une part, la coopération est nécessaire au bon déroulement des activités professionnelles et, d'autre part, les signaux de marquage d'appartenance culturelle fonctionnent pour ainsi dire " à l'envers », l'humour et le rire permettent d'insérer du jeu au sein d'articulations sociales mises à rude épreuve. En riant avec les autres employés à propos de stéréotypes propres à sa culture d'appartenance, on (ré)affirme sa position de collaborateur au sein du collectif des employés. En participant à un système de relations à plaisanterie, on s'assure que les différents signes d'appartenance culturels ne sont, dans la situation présente, en rien menaçants et ne sont pas à prendre au sérieux.

Si nous nous sommes essentiellement intéressés dans cet article au « nous professionnel » par rapport au « nous d'origine », les différents nous qui coexistent à l'usine sont en fait plus nombreux et forment un ensemble plus complexe qu'une simple opposition binaire. A un niveau macro, à l'échelle de la vie à l'usine, il s'agit de créer un « nous collègues », regroupant tous les individus en faisant fi de leurs caractéristiques fortement hétérogènes (âge, origine socioculturelle, langue maternelle etc.). A l'intérieur de ce groupe, coexistent et cohabitent des groupes de type plus micro qui regroupent leurs membres en fonction de critères communs tels que celui d'être « non-suisse » ou encore d'être des « compatriotes ». C'est l'articulation positive nécessaire de ces différents " nous » au sein d'un " nous collègues » partiellement contraint par le contexte et le travail qui constitue le cœur et l'intérêt de notre recherche. L'élaboration, le maintien, la négociation de ces relations se réalisent de manière quotidienne; elles se définissent et se modifient au cours des interactions banales de la vie en usine. Considérant par ailleurs que les appartenances à un " nous d'origine », autant qu'à un «nous professionnel », sont des éléments constitutifs de l'identité, notre propos s'inscrit dans une conception éminemment relationnelle du sujet ${ }^{11}$.

Dans ce contexte, le rire semble agir tout spécifiquement en « lubrifiant social » des relations (pour reprendre la formule de Morreall 1991 : 370) potentiellement conflictuelles dans le cadre de relations intergroupes contraintes. A des conditions de vie spécifiques, amenant une forme de proximité contrainte et une nécessité de faire groupe correspondent des rires et des énoncés humoristiques spécifiques. C'est le cas des vannes à caractère raciste dans un milieu ouvrier fortement multiculturel et, plus largement, celui des relations à plaisanterie. Ainsi, dans ces situations de travail en milieu interculturel, les rires résultent des tensions induites « automatiquement » par la fréquentation d'habitus culturels différents, associée à la nécessité de se coordonner les uns avec les autres afin d'organiser le travail en commun.

11 Pour une présentation détaillée de cette conception, voir Kaufmann Laurence et Krzysztof Skuza, 2008. 


\section{Références citées}

Allès, Elisabeth, 2003. « Notes sur quelques relations à plaisanteries entre villages hui (Chinois musulmans) et han du Henan », Perspectives chinoises 78.

Bachorowski, Jo-Anne et Michael J. Owren, 2001. « Not All Laughs are Alike : Voiced but not Unvoiced Laughter Readily Elicits Positive Affect », Psychological Science 12 (3) : 252-257.

Bradney, Pamela, 1957. « The Joking Relationship in Industry », Human Relations 10 : 179-187.

Bernhard, Helen, Urs Fischbacher et Ernst Fehr, 2006. « Parochial Altruism in Humans », Nature 442 (7105) : 912-91.

Brackelaire, Jean-Luc, 1993. « Changer pour rire. Les relations de plaisanterie des Tarahumaras : figure et mesure du Changement », Anthropologie et Sociétés 17 (3) : 125-140.

Coser Laub, Rose, 1960. « Laughter among Colleagues », Psychiatry 23 (1) : 81-95.

Diallo, Youssouf, 2006. "Identités et relations de plaisanterie chez les Peuls de l'ouest du Burkina Faso », Cahiers d'études africaines 4 (184) : 779-794.

Dunbar, Robin I. M., 1996. Grooming, Gossip and the Evolution of Language. Harvard University Press.

Dwyer, Tom, 1991. « Humor, Power, and Change in Organizations », Human Relations 44 (1) : 1-19.

Favret-Saada, Jeanne, 2007. Comment produire une crise mondiale avec douze petits dessins. Paris : Les Prairies ordinaires.

Fay, Claude, 2006. « Sang, lait, distance et plaisanterie », Cahiers d'études africaines 4 (184) : 755-778.

Fehr, Ernst et Urs Fischbacher, 2004. " Social Norms and Human Cooperation », Trends Cogn Sci (Reg), 8 (4) : 185-190.

Fehr, Ernst et Herbert Gintis, 2007. «Human Motivation and Social Cooperation : Experimental and Analytical Foundation », The Annual Review of Sociology 33 : 43-64.

Frisch-Gauthier, Jacqueline, 1961. «Le rire dans les relations de travail », Revue de sociologie française $4(2): 292-303$.

Gil-White, Francisco, 2005. « How Conformism Creates Ethnicity Creates Conformism (And Why this Matters to Lots of Things) », The Monist 88 (2) : 189-237.

Hochschild, Arlie R., 2003. «Travail émotionnel, règles de sentiments et structure sociale », Travailler $1(9): 19-49$.

Kaufmann, Laurence, 2002. "La prédication "nostrologique”. Quelques réflexions sur la nature du politique », Revue Européenne des Sciences Sociales XL (24) : 283-308.

Kaufmann, Laurence et Krzysztof Skuza, 2008. " Esquisse d'une sociologie à la première personne », in A. Giovannoni et J. Guilhaumou (éds), Histoire et subjectivation, 57-100. Paris : Kimé.

Labouret, Henri, 1929. "La parenté par plaisanteries en Afrique Occidentale », Africa : Journal of the International African Institut 3 (2) : 244-254.

Lynch, Owen, 2010. « Cooking With Humor : In-Group Humor in Social Organization », Humor 23 (2) : 127-159.

Mehu, Marc et Robin I. M. Dunbar, 2008. « Relationship between Smiling and Laughter in Humans (Homo sapiens) : Testing the Power Asymmetry Hypothesis », Folia Primatologica 79 (5) : 269-280.

Moreau, Reginald E., 1944. « Joking Relationships in Tanganyika », Africa : Journal of the International African Institute 14 (7) : 386-400.

Morreall, John, 1991. « Humor and Work », Humor 4 (3/4) : 359-373.

Owren, Michael J. et Jo-Anne Bachorowski, 2003. «Reconsidering the Evolution of Communication : the Case of Laughter », Journal of Nonverbal Behavior 27 (3) : 183-200. 
Panksepp, Jaak, 2007. « Neuroevolutionary Sources of Laughter and Social Joy : Modeling Primal Human Laughter in Laboratory Rats », Behavioural Brain Research 182 (2) : 231-244.

Parry, Jonathan P., 2002. « Du bagne des champs aux riantes usines. Le travail dans une entreprise sidérurgique indienne », Terrain $39: 121-140$.

Paulme, Denise, 1939. «Parenté à plaisanteries et alliance par le sang en Afrique occidentale », Africa : Journal of the International African Institute 4 (12) : 433-444.

Provine, Robert R., 2003. Le rire, sa vie, son ouvre : le plus humain des comportements expliqué par la science. Paris : Robert Laffont.

Provine, Robert R. et Karen Emmorey, 2006. « Laughter Among Deaf Signers », Journal of Deaf Studies and Deaf Education 11 (4) : 403-409.

Radcliffe-Brown, Alfred Reginald, 1968. Structure et fonction dans la société primitive. Paris : Editions de Minuit.

Ratouis, Olivier et Martin Baumeister, 2011. « Rire en ville. Rire de la ville. L'humour et le comique comme objets pour l'histoire urbaine contemporaine », Histoire urbaine 2 (31) : 5-18.

Servais, Véronique, 1999. «Autour du chat de Cheshire et de son sourire. Approche comparative du rire et du sourire », L'Homme 39 (150) 157-175.

Sissao, Alain Joseph, 2004. «Ethnicité et culture : l'Alliance à plaisanterie comme forme de culture ciment entre les ethnies au Burkina Faso », Associations transnationales 4 : 269-280.

Smoski, Moria J. et Jo-Anne, A. Bachorowksi, 2003. « Antiphonal Laughter in Developing Friendships », Annals of the New York Academy of Sciences 1000 (1) : 300-303. 
